\title{
Generic appearance of objective results in quantum measurements
}

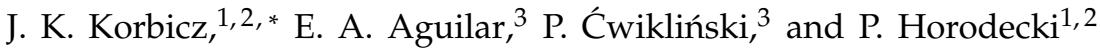 \\ ${ }^{1}$ Faculty of Applied Physics and Mathematics, Gdańsk University of Technology, 80-233 Gdańsk \\ ${ }^{2}$ National Quantum Information Centre in Gdańsk, 81-824 Sopot, Poland \\ ${ }^{3}$ Faculty of Mathematics, Physics and Informatics University of Gdańsk, 80-952 Gdańsk, \\ National Quantum Information Centre in Gdańsk, 81-824 Sopot, Poland
}

(Dated: March 3, 2022)

\begin{abstract}
Measurement is of central interest in quantum mechanics as it provides the link between the quantum world and the world of everyday experience. One of the features of the latter is its robust, objective character, contrasting the delicate nature of quantum systems. Here we analyze in a completely model-independent way the celebrated von Neumann measurement process, using recent techniques of information flow, studied in open quantum systems. We show the generic appearance of objective results in quantum measurements, provided we macroscopically coarse-grain the measuring apparatus and wait long enough. To study genericity, we employ the widely-used Gaussian Unitary Ensemble of random matrices and the Hoeffding inequality. We derive generic objectivization timescales, given solely by the interaction strength and the systems' dimensions. Our results are manifestly universal and are a generic property of von Neumann measurements.
\end{abstract}

PACS numbers: 05.30.-d 03.67.-a 03.65.Yz

Keywords: Quantum Measurement Problem, Objectivity, Random Matrix Theory

Understanding quantum measurements has been one of the central problems of quantum theory since its beginning [1, 2]. It not only provides the crucial link between the theory and experiment, the micro- and macro-worlds, but is at the heart of the modern quantum technologies (see e.g. [3]). The fundamental measurement theory dates back to von Neumann [4] and since then has been further developed in various directions, e.g. the decoherence theory [5, 6]. To be readable, measurement results must inevitably be encoded into macroscopic degrees of freedom and one of the crucial features expected from a good measurement process is an objective character of the results: They can be read out by arbitrary many observers and without causing any disturbance by the mere read-out. This has been realized as early as in 1929 by Mott [7]. Achieved in well engineered measurements by a proper coupling to macroscopic degrees of freedom, it is not at all obvious if such a situation is a generic feature of a quantum measurement process with a macroscopic recording.

In a broader context of open quantum systems [5, 6], this may be seen as a question about how information flows from the system to its environment. Pioneering research along this direction has been undertaken under the quantum Darwinism idea [8], arguing that in some situations (see e.g. [9, 10]) perfect information about the system can be redundantly stored in the environment and becomes effectively classical [11] and objective. The generic character of some of the quantum Darwinism features was shown in [12] and the universality of decoherence was shown on short time-scales in [13-16]. A further step was recently made in [17, 18]

*Electronic address: jkorbicz@mif.pg.gda.pl by formulating information flow and objectivity in the fundamental language of quantum states with the introduction of the, so called, Spectrum Broadcast Structures (SBS's). The latter has been proven to be a useful tool allowing to obtain novel results in some of the emblematic models of decoherence [17, 19,-21]. Finally, questions of genericity have traditionally been the domain of statistical mechanics and thermodynamics (see e.g. [22-24]). Phrased in this language, we may ask to what form a generic state equilibrates during a von Neumann measurement.

In this communication we study information flow during a von Neumann measurement process with a macroscopic (in a sense of a number of degrees of freedom) measuring apparatus. Applying random matrix theory techniques [25, 26], we show that generically the post-measurement state approaches, after a coarse-graining, a form carrying almost perfect, multiple records of the measurement result, thus making the latter objective. To study genericity, we use a properly structured Gaussian Unitary Ensemble (GUE) [25, 26]. Since the seminal works of Wigner and Dyson on statistics of various experimentally observed spectra, it has been the basic choice for random Hamiltonians due to its universality and agreement with the experiment [25, 26]. The apparatus is assumed to be noisy, with the initial state distributed according to some physically motivated measures of mixed states [27]. For largedimensional measured systems, we provide estimates on the time-scale of the objectivization process. Since the only assumptions we make concern the genericity measures, our results are manifestly universal and apply to the whole class of von Neumann measurements, thus showing a generic and robust character of the emergence of objectivity. It is a bit of a surprise that this property of von Neumann measurements was so 
far tacitly assumed (see e.g. [28]) but never, to our best knowledge, derived.

Measurements with compound apparatuses.- (cf. [17]) We consider a $d_{S}$-dimensional quantum system $S$ simultaneously measured by a collection of $N$ measuring apparatuses/environments $E_{1}, \ldots, E_{N}$, each of dimension $d$, representing a macroscopic measuring device. The apparatuses are assumed to be individually coupled to the system through a general von Neumanntype interaction, so strong that the self Hamiltonians of the system and the apparatuses can be neglected (the quantum measurement limit) [4]:

$$
\hat{H}_{\text {total }} \approx \hat{H}_{\text {int }}=\hat{A} \otimes \sum_{k=1}^{N} \hat{B}_{k}
$$

where $\hat{A}$ is the measured observable (assumed nondegenerate) and the $\hat{B}_{k}$ are some general measuring observables. This leads to the evolution (setting $\hbar=1$ ) $\hat{U} \equiv \mathrm{e}^{-i t \hat{H}_{i n t}}=\sum_{a}|a\rangle\langle a| \otimes \bigotimes_{k=1}^{N} \mathrm{e}^{-i a \hat{B}_{k} t}$, where $\hat{A}=$ $\sum_{a=1}^{d_{S}} a|a\rangle\langle a|$. Our main object of study is a partially reduced state $\rho_{S: E_{o b s}}$, with a fraction $E_{u n o}$ of size $N_{u n o}$ of unobserved subsystems traced out. This represents an inevitable loss of information during a measurement.Assuming $\rho_{S E}(0)=\rho_{0 S} \otimes \bigotimes_{k=1}^{N} \rho_{0 k}$ we obtain:

$$
\begin{aligned}
& \rho_{S: E_{o b s}}(t)=\sum_{a} p_{a}|a\rangle\langle a| \otimes \bigotimes_{k=1}^{N_{o b s}} \rho_{a k}(t)+\sum_{a} \sum_{a^{\prime} \neq a} c_{a a^{\prime}} \\
& \times\left\{\prod_{k=1}^{N_{\text {uno }}} \operatorname{Tr}\left[\mathrm{e}^{-i\left(a-a^{\prime}\right) \hat{B}_{k} t} \rho_{0 k}\right]\right\}|a\rangle\left\langle a^{\prime}\right| \bigotimes_{k=1}^{N_{o b s}} \mathrm{e}^{-i a \hat{B}_{k} t} \rho_{0 k} \mathrm{e}^{i a^{\prime} \hat{B}_{k} t},
\end{aligned}
$$

where $p_{a} \equiv\left\langle a\left|\rho_{0 S}\right| a\right\rangle, \quad c_{a a^{\prime}} \equiv\left\langle a\left|\rho_{0 S}\right| a^{\prime}\right\rangle, \rho_{a k}(t) \equiv$ $\mathrm{e}^{-i a \hat{B}_{k} t} \rho_{0 k} \mathrm{e}^{i a \hat{B}_{k} t}, N_{\text {uno }}+N_{\text {obs }}=N$. We define the decoherence factor for the unobserved fraction $E_{\text {uno }}$ :

$$
\Gamma_{a a^{\prime}}^{u n o}(t) \equiv \prod_{k=1}^{N_{u n o}}\left|\operatorname{Tr}\left[\mathrm{e}^{-i\left(a-a^{\prime}\right) \hat{B}_{k} t} \rho_{0 k}\right]\right|^{2}
$$

If for all $a \neq a^{\prime}$ : i) $\Gamma_{a a^{\prime}}^{u n o}(t)=0$, i.e. decoherence takes place, and ii) $\rho_{a k}(t) \perp \rho_{a^{\prime} k}(t)$, i.e. $\rho_{a k}(t)$ are perfectly distinguishable, then we say that $\rho_{S: E_{n h s}}(t)$ is of a Spectrum Broadcast Structure (SBS) [17--19] with respect to (w.r.t.) the basis $|a\rangle$ (this context-dependence is of a fundamental importance, see e.g. [29]), defined as [30]:

$$
\rho_{S B S}=\sum_{a} p_{a}|a\rangle\langle a| \otimes \rho_{a} \otimes \cdots \otimes \rho_{a}, \rho_{a} \perp \rho_{a^{\prime} \neq a} .
$$

The basis $|a\rangle$ becomes then the, so-called, pointer basis in which the system has decohered and the result of the measurement, $a$, appearing with the probability $p_{a}$, is stored in the measuring setup in many, perfect copies. Crucially, their readouts, through projections on the supports of $\rho_{a k}(t)$, will not disturb (on average) the joint state $\rho_{S: E_{o b s}}(t)$. This leads to a form of objectivity of the measurement result: It can be read out by multiple observers without disturbing neither the (decohered) system nor themselves [8, 17, 18]. In quantum-information terms, this objectivization process is a weaker form of quantum state broadcasting [31, 32]. We can thus reformulate the original question as: Are SBS's generic for the interactions (1)? To address it, we introduce an ensemble of random Hamiltonians of the form (1) and random initial conditions $\rho_{0 k}$. We then estimate the average trace distance between the actual state (2) and an ideal SBS in the following steps: i) calculate the averages over $\hat{B}_{k}$ of the decoherence factor (3) and the, so called, super-fidelity bound for the states $\rho_{a k}(t)$; ii) average them over $\rho_{0 k}$; iii) coarse-grain the apparatus; iv) further average over $\hat{A}$; v) use the central result of [21] to bound the average distance and show that it vanishes in the macroscopic limit. We then use the concentration inequality of Hoeffding [33], following from the classical Chernoff bound, to show genericity.

The coarse-graining is one of the crucial steps. As we will show, on the microscopic level of the individual apparatuses, the residual noise is too strong to allow a SBS formation even asymptotically. This can be overcome if we group the $N_{o b s}$ observed apparatuses into fractions scaling with $N$ (called macrofractions) and pass to the thermodynamic limit $N \rightarrow \infty$ [17]. The number $\mathcal{M}$ of such groups (assumed for simplicity equal) is irrelevant, provided their sizes $N_{m a c} \equiv N_{o b s} / \mathcal{M}$ satisfy $N_{m a c} \sim N$. These macrofractions may be understood as reflecting some detection threshold, e.g. a minimum bunch of photons the eye can detect.

Randomizing measurement Hamiltonians.- We introduce an ensemble of random measurement Hamiltonians (1) using the widely-used Gaussian Unitary Ensemble [25, 26] in the following way (cf. [34, 35]): i) $\hat{B}_{k}$ are independently, identically distributed (i.i.d.) according to a GUE with a scale factor $\eta_{E}$; ii) $\hat{A}$ is distributed according to its own GUE with a scale factor $\eta_{S}$. We recall that the GUE measure is defined as:

$$
\mathrm{d} \mu_{\text {gue }}(\hat{H})=\frac{1}{Z} \mathrm{e}^{-\frac{\eta}{2} \sum_{i} \lambda_{i}^{2}} \prod_{i<j}\left(\lambda_{i}-\lambda_{j}\right)^{2} \mathrm{~d} \lambda \mathrm{d} \hat{\mathcal{U}},
$$

with $Z$ the normalization, $\lambda_{i}$ the eigenvalues, $\eta$ a scale factor, and $\mathrm{d} \hat{\mathcal{U}}$ the Haar measure on the unitary group.

The simultaneous vanishing of the decoherence factor (3) and of the generalized overlaps [31, 36] $F_{a a^{\prime}} \equiv$ $F\left(\rho_{a}, \rho_{a^{\prime}}\right) \equiv\left(\operatorname{Tr} \sqrt{\sqrt{\rho_{a}} \rho_{a^{\prime}} \sqrt{\rho_{a}}}\right)^{2}$ for all $a \neq a^{\prime}$ has so far been used to witness a SBS formation [17, 21]. The latter function is however complicated and here we will use the so-called super-fidelity bound [37] $F(\rho, \sigma) \leq$ $G(\rho, \sigma) \equiv \operatorname{Tr}(\rho \sigma)+\sqrt{\left(1-\operatorname{Tr} \rho^{2}\right)\left(1-\operatorname{Tr} \sigma^{2}\right)}$ (although we note that it is not tight if both states are mixed, as e.g. for $\rho \perp \sigma, G(\rho, \sigma) \neq 0)$, which here reads:

$$
G\left(\rho_{a}(t), \rho_{a^{\prime}}(t)\right)=\operatorname{Tr}\left(\rho_{a}(t) \rho_{a^{\prime}}(t)\right)+S_{l i n}\left(\rho_{0}\right) \equiv G_{a a^{\prime}}(t),
$$


where $S_{\text {lin }}\left(\rho_{0}\right) \equiv 1-\operatorname{Tr} \rho_{0}^{2}$ is the linear entropy of the initial state of an individual apparatus.

We now average (36) over the interaction and the initial conditions. We first average over $\left\{\hat{B}_{k}\right\}$, fixing the levels $a, a^{\prime}$ of $\hat{A}$. We have:

$$
\left\langle\Gamma_{a a^{\prime}}^{u n o}(t)\right\rangle_{\left\{\hat{B}_{k}\right\}}=\prod_{k=1}^{N_{u n o}}\left\langle\left|\operatorname{Tr}\left[\mathrm{e}^{-i\left(a-a^{\prime}\right) \hat{B}_{k} t} \rho_{0 k}\right]\right|^{2}\right\rangle_{\hat{B}_{k^{\prime}}}
$$

since $\hat{B}_{k}$ are i.i.d. Modulo $\rho_{0 k}$, all the factors are identical and we calculate the average over a single $\hat{B}_{k}$, dropping the index $k$ for simplicity. Performing the Haar integration first ([38], Section IA) and then the eigenvalue one ([38], Section IIB), we obtain [39]:

Result 1. The GUE averages of the single environment decoherence and super-fidelity factors read:

$$
\begin{aligned}
& \left\langle\Gamma_{a a^{\prime}}(t)\right\rangle=\frac{1+\operatorname{Tr} \rho_{0}^{2}}{d+1}+\left\langle f_{t}(\boldsymbol{a}, \boldsymbol{\lambda})\right\rangle \frac{2\left(d-\operatorname{Tr} \rho_{0}^{2}\right)}{d\left(d^{2}-1\right)}, \\
& \left\langle G_{a a^{\prime}}(t)\right\rangle=S_{\text {lin }}\left(\rho_{0}\right)+\frac{1+\operatorname{Tr} \rho_{0}^{2}}{d+1} \\
& +\left\langle f_{t}(\boldsymbol{a}, \boldsymbol{\lambda})\right\rangle \frac{2\left(d \operatorname{Tr} \rho_{0}^{2}-1\right)}{d\left(d^{2}-1\right)}
\end{aligned}
$$

with $f_{t}(\boldsymbol{a}, \boldsymbol{\lambda}) \equiv \sum_{m} \sum_{n>m} \cos \left[\left(a-a^{\prime}\right)\left(\lambda_{n}-\lambda_{m}\right) t\right]$ and:

$$
\begin{aligned}
& \left\langle f_{t}(\boldsymbol{a}, \boldsymbol{\lambda})\right\rangle=p\left(d, \tilde{\Delta}_{t}\right) \mathrm{e}^{-\tilde{\Delta}_{t}^{2}} \\
& p\left(d, \tilde{\Delta}_{t}\right) \equiv \\
& \sum_{n} \sum_{m>n}\left[L_{n}^{(0)}\left(\tilde{\Delta}_{t}^{2}\right) L_{m}^{(0)}\left(\tilde{\Delta}_{t}^{2}\right)-\frac{n !}{m !} \tilde{\Delta}_{t}^{2(m-n)}\left[L_{n}^{(m-n)}\left(\tilde{\Delta}_{t}^{2}\right)\right]^{2}\right]
\end{aligned}
$$

where $\tilde{\Delta}_{t} \equiv\left(a-a^{\prime}\right) t / \sqrt{\eta_{E}}$ and $L_{n}^{(m)}$ are the associated Laguerre polynomials.

The above results are exact. Although the average $\left\langle f_{t}(\boldsymbol{a}, \boldsymbol{\lambda})\right\rangle$ with the GUE eigenvalue distribution $P_{\text {gue }}(\boldsymbol{\lambda})$ involves only the two-point correlation function [25]: $\quad R_{2}\left(\lambda_{1}, \lambda_{2}\right) \equiv d ! /(d-$ $2) ! \int \cdots \int \mathrm{d} \lambda_{3} \cdots \mathrm{d} \lambda_{d} P_{\text {gue }}\left(\lambda_{1}, \ldots, \lambda_{d}\right)$ (due to the symmetry), and the large- $d$ asymptotics of $R_{2}\left(\lambda_{1}, \lambda_{2}\right)$ are well known [25], they are of no use here. One can show that [25]: $R_{2}\left(\lambda_{1}, \lambda_{2}\right)=K_{d}\left(\lambda_{1}, \lambda_{1}\right) K_{d}\left(\lambda_{2}, \lambda_{2}\right)-$ $\left[K_{d}\left(\lambda_{1}, \lambda_{2}\right)\right]^{2}, K_{d}\left(\lambda_{1}, \lambda_{2}\right) \equiv \sum_{j=0}^{d-1} \phi_{j}\left(\lambda_{1}\right) \phi_{j}\left(\lambda_{2}\right)$, with $\phi_{j}(\lambda)$ the oscillator wave-functions, and while the first term approaches the Wigner semicircle distribution, integrable with $f_{t}(\boldsymbol{a}, \boldsymbol{\lambda})$, the second term approaches a function of $\left|\lambda_{1}-\lambda_{2}\right|$ only [25] and makes the integral divergent. That is the integration with $f_{t}(\boldsymbol{a}, \boldsymbol{\lambda})$ and the large- $d$ limit are not interchangeable here.

Both (8.9) depend on $\rho_{0}$ only through its purity $\operatorname{Tr} \rho_{0}^{2}$ and we can use the known results of generic state purity to effectively get rid of the initial state dependence. Although there is no canonical choice of a measure over mixed states, there are several popular ones e.g. the
Hilbert-Schmidt and the Bures measures [27] giving:

$$
\left\langle\operatorname{Tr} \rho_{0}^{2}\right\rangle_{H S}=\frac{2 d}{d^{2}+1},\left\langle\operatorname{Tr} \rho_{0}^{2}\right\rangle_{B u}=\frac{5 d^{2}+1}{2 d\left(d^{2}+2\right)} .
$$

Especially the Bures measure is physically important as it: i) is directly connected to quantum metrology [40]; ii) reproduces the correct measure for pure states. In what follows we will assume that $\rho_{0 k}$ are i.i.d. with one of the above measures and are averaged over.

Residual noise and coarse-graining.- As $p\left(d, \tilde{\Delta}_{t}\right)$ is an even polynomial of degree $2(2 d-3),(10)$ implies that the time dependent part in (8, 9) decays for any fixed $d$ and a gap $\left|a-a^{\prime}\right| \neq 0$ with a characteristic time $\tau_{a a^{\prime}} \equiv\left|a-a^{\prime}\right|^{-1} \sqrt{\eta_{E} /(d+1)}$ ([38], Section II). The remaining constant terms: A common one of the order $O(1 / d)$ (cf. (12)), called "white noise", and additionally $\left\langle S_{\text {lin }}\left(\rho_{0}\right)\right\rangle$ in (9). The latter, arising from the non-tight bound (6), is intuitively understood - the noisier the apparatus is initially, the lesser information, measured by the state distinguishability, it can accumulate. These factors, reflecting residual background fluctuations in the ensemble, pertain to a single apparatus and prevent a SBS formation. However, coming back to (3), using (7) and (12), we actually obtain an exponential decay with $N_{\text {uno }}$ of the collective decoherence factor:

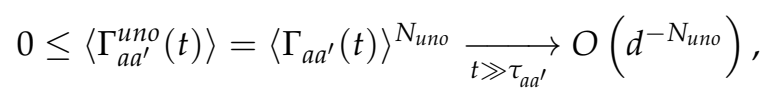

showing that for a large local dimension $d$ and/or large unobserved fraction $N_{\text {uno }}$, measurement dynamics (1) generically leads to decoherence (cf. [13]). The same step can be preformed on the observed fraction too [17]: We group the $N_{o b s}$ observed apparatuses into $M$ groups of $N_{\text {mac }}$ each, described by states $\rho_{a}^{m a c}(t) \equiv$ $\bigotimes_{k \in \text { mac }} \rho_{a k}(t)$. Due to the factorization of fidelity w.r.t. the tensor product and the i.i.d. property, the resulting super-fidelity bound (6) for the group also decays (cf. (12):

$$
0 \leq\left\langle F_{a a^{\prime}}^{m a c}(t)\right\rangle \leq\left\langle G_{a a^{\prime}}(t)\right\rangle^{N_{m a c}} \underset{t \gg \tau_{a a^{\prime}}}{\longrightarrow} O\left(\mathrm{e}^{-\frac{N_{m a c}}{d}}\right) .
$$

If both $N_{\text {uno }}, N_{\text {mac }}$ scale with $N$, 13]14 can be made small in the macroscopic/thermodynamic limit $N \rightarrow$ $\infty$. Crucially, increasing $d$ alone is not enough-it damps the white noise, but $\left\langle S_{\operatorname{lin}}\left(\rho_{0}\right)\right\rangle \simeq 1-O(1 / d)$ by (12).

Generic post measurement state and objectivity.Results 8. 9) still depend on the $\hat{A}^{\prime}$ s level differences $\left|a-a^{\prime}\right|$. To study a completely general behavior, a further averaging of $\left\langle\Gamma_{a a^{\prime}}^{u n o}(t)\right\rangle,\left\langle G_{a a^{\prime}}^{m a c}(t)\right\rangle$ over the levels $a, a^{\prime}$ should be performed with the corresponding twopoint correlation function $R_{2}\left(a, a^{\prime}\right)$ (the average is independent of the labels $a, a^{\prime}$ due to the symmetry). The resulting integrals are intractable, but from (8) A52) they will eventually reach the noise-floor (see Fig. 1). Lower bounds on the relevant timescales can be obtained from 


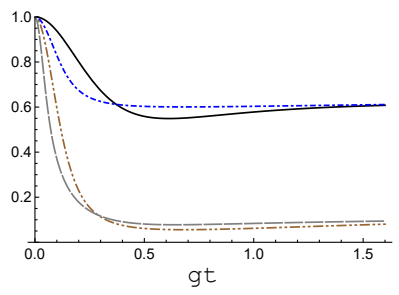

(a) $N_{\text {uno }}=1$

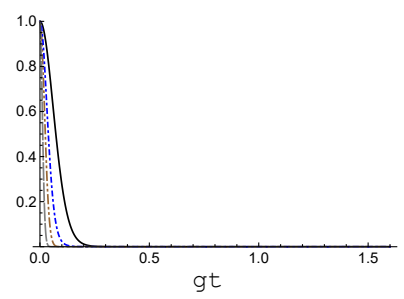

(c) $N_{\text {uno }}=20$

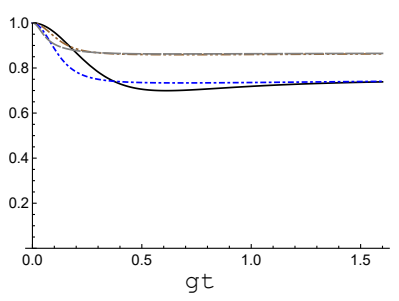

(b) $N_{m a c}=1$

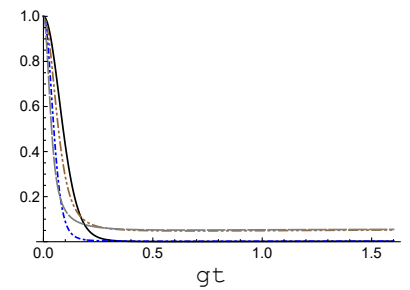

(d) $N_{m a c}=20$
FIG. 1: (Color online). Time dependence of the exact full averages of the decoherence factor (a),(c) and the super-fidelity (b),(d) for different dimensions and macrofraction sizes. The two-point correlation function $R_{2}\left(a, a^{\prime}\right)$ averages of the exact solutions 8, 9] were used, with $\left\langle\operatorname{Tr} \rho_{0}^{2}\right\rangle_{B u}$ from (12). Different combinations of $\left\{d_{S}, d\right\}$ are plotted: $\{2,2\}$ solid black; $\{2,10\}$ brown dot-dot-dash; $\{10,2\}$ blue dot-dash; $\{10,10\}$ gray long-dash. The time is in the the units of the interaction strength $g \equiv 1 / \sqrt{\eta_{S} \eta_{E}}$ [41].

a short-time analysis ([38], Section II), giving for the decoherence and the superfidelity respectively:

$$
\begin{gathered}
\tau_{\text {dec }} \equiv\left[8 g^{2} N_{\text {uno }} d_{S}\left(d-\left\langle\operatorname{Tr} \rho_{0}^{2}\right\rangle\right)\right]^{-\frac{1}{2} d \gg 1} \frac{g^{-1}}{\sqrt{N_{\text {uno }} d_{S} d}}, \\
\tau_{f i d} \equiv\left[8 g^{2} N_{\text {mac }} d_{S}\left(d\left\langle\operatorname{Tr} \rho_{0}^{2}\right\rangle-1\right)\right]^{-\frac{1}{2} d \gg 1} \frac{g^{-1}}{\sqrt{N_{\text {mac }} d_{S}}} .
\end{gathered}
$$

Here $g^{-1} \equiv \sqrt{\eta_{S} \eta_{E}}$ is the effective interaction time-scale and $d_{S}$ is the system dimension. We see a characteristic separation of time-scales: From (12), $\tau_{f i d} \sim \sqrt{d} \tau_{\text {dec }}$ for the same macrofraction sizes. Thus, on average, it takes longer to accumulate information in the apparatus than to decohere the system [17, 20]. Combining (15) with Result 1 and (13|14) we arrive at (cf. [42]):

Result 2. The interaction and initial state averages satisfy:

$$
\begin{aligned}
& \left\langle\left\langle\Gamma^{u n o}(t)\right\rangle\right\rangle \underset{t \gg \tau_{\text {dec }}}{\longrightarrow} O\left(\mathrm{e}^{-N_{\text {uno }} \log d}\right), \\
& \left\langle\left\langle G^{\text {mac }}(t)\right\rangle\right\rangle \underset{t \gg \tau_{\text {fid }}}{\longrightarrow} O\left(\mathrm{e}^{-\frac{N_{\text {mac }}}{d}}\right) .
\end{aligned}
$$

Next crucial step is to use the result of [21] estimating an optimal trace distance between (2) and an ideal SBS state on the coarse-grained level of macrofractions:

$$
\begin{aligned}
& \epsilon_{S B S}(t) \equiv \frac{1}{2}\left\|\rho_{S: E_{o b s}}(t)-\rho_{S B S}\right\|_{\operatorname{Tr}} \\
& \leq \sum_{a} \sum_{a^{\prime} \neq a}\left[\left|c_{a a^{\prime}}\right| \sqrt{\Gamma_{a a^{\prime}}^{u n o}(t)}+\sqrt{p_{a} p_{a^{\prime}}} \sum_{m a c} \sqrt{F_{a a^{\prime}}^{m a c}(t)}\right]
\end{aligned}
$$

Using $p_{a},\left|c_{a a^{\prime}}\right| \leq 1,\langle\sqrt{f}\rangle \leq \sqrt{\langle f\rangle}$ for $f \geq 0$, the superfidelity bound, and the Result 2 , estimation 18 gives:

Result 3. Averaged over all the von Neumann measurements (1) and the initial conditions, the optimal distance of the actual state (2) to an ideal SBS state satisfies:

$$
\left\langle\left\langle\epsilon_{S B S}(t)\right\rangle\right\rangle \underset{t \gg \tau_{S B S}}{\longrightarrow} O\left[d_{S}^{2}\left(\mathrm{e}^{-\frac{N_{\text {uno }}}{2} \log d}+\mathcal{M} \mathrm{e}^{-\frac{N_{m a c}}{2 d}}\right)\right]
$$

where $\tau_{S B S}$ is the larger of 15$)$ and $\mathcal{M}$ is the number of macrofractions into which the observed degrees of freedom of the apparatus are coarse-grained.

Finally, since $0 \leq \epsilon_{S B S}(t) \leq 1$ is a bounded random variable for any $t$, it follows from the Hoeffding inequality [33] that: $P\left[\left|\epsilon_{S B S}(t)-\left\langle\left\langle\epsilon_{S B S}(t)\right\rangle\right\rangle\right| \geq \delta\right] \leq$ $2 \mathrm{e}^{-2 \delta^{2}}$ for any $\delta \geq 0$. This, together with Result 3 shows the genericity of the SBS formation for large enough apparatuses and long enough times.

Conclusions.-A measurement is an inevitable part of any quantum experiment and the results must inevitably be encoded into macroscopic degrees of freedom and become effectively classical for us to read. This in particular entails becoming objective. We studied this process using the general von Neumann measurement scheme (1) with a macroscopic measuring apparatus. A huge amount of degrees of freedom $(N \sim$ $10^{23}$ ) makes it in practice impossible to observe them all and to control each individual coupling. A way to model this physical situation is to introduce some randomness and ask questions about genericity. We did it in two steps: First we randomized the measurement device side (the observables and the generically noisy initial states) and showed that after including the inevitable losses and macroscopic coarse-graining, a post measurement state approaches the so called SBS form asymptotically for almost any initial conditions and couplings. The timescales of this process depended on the spectral gap of the measured observable on the system side. Afterwards, to get rid of this dependence, we went beyond a single experiment scenario, randomizing the measured observable too. An interesting aspect of that second randomization is that this may be viewed as a quite natural assumption of any quantum system interacting with many objects. Indeed it is natural to assume that it interacts with each of the objects with some fixed, yet different than with the others, way. Since there are many objects, then the averaging effect comes from that variety of the interactions and can be viewed as a self-averaging of the system plus environment com- 
plex. This led to our central result: Almost any quantum measurement produces objective outcomes on the macroscopic level on the timescale given by the larger of (15). This is a universal, model-independent result.

We believe one can go beyond the genericity notion used here (Hoeffding inequality) and show the concentration of measure phenomenon, e.g. by combining the results for the Wigner-type matrices [43] with the methods of [22, 42]. Another possible future direction is to go beyond the quantum measurement limit and consider non-trivial dynamics of the system and the measuring device. A candidate tool for such an analysis already exists in the form of dynamical SBS [19].

\section{Acknowledgements}

This work was carried out at the National Quantum Information Centre in Gdańsk. We thank J. Wehr, P. Grangier, M. Horodecki, and R. Horodecki for discussions. JKK and PH acknowledge the financial support of the John Templeton Foundation through the grant ID \#56033. EAA is supported by the grant Sonata Bis 2014/14/E/ST2/00020 from National Science Centre. PĆ thanks the Seventh framework programme EU grant RAQUEL No 323970 and the grant PRELUDIUM 2015/17/N/ST2/04047 from National Science Centre.
[1] N. Bohr, in The Library of Living Philosophers, Volume 7. Albert Einstein: Philosopher-Scientist, edited by P. A. Schilpp (Open Court, 1949), pp. 199-241.

[2] W. Heisenberg, Philosophic problems of nuclear science, Fawcett premier book (Faber and Faber, 1952).

[3] H. M. Wiseman and G. J. Milburn, Quantum Measurement and Control (Cambridge University Press, Leiden, 2009).

[4] J. von Neumann, Mathematical Foundations of Quantum Mechanics (Princeton Univ. Press, Princeton, N. J., 1955).

[5] M. A. Schlosshauer, Decoherence and the Quantum-ToClassical Transition (Springer-Verlag, Berlin, 2007).

[6] E. Joos, Decoherence and the Appearance of a Classical World in Quantum Theory, Physics and astronomy online library (Springer, 2003).

[7] N. Mott, Proc. R. Soc. A 126, 79 (1929).

[8] W. H. Żurek, Nat. Phys. 5, 181 (2009).

[9] C. J. Riedel and W. H. Żurek, Phys. Rev. Lett. 105, 020404 (2010).

[10] R. Blume-Kohout and W. H. Żurek, Phys. Rev. Lett. 101, 240405 (2008).

[11] H. Ollivier, D. Poulin, and W. H. Żurek, Phys. Rev. Lett. 93, 220401 (2004).

[12] F. G. S. L. Brandão, M. Piani, and P. Horodecki, Nat. Commun. 6 (2015).

[13] D. Braun, F. Haake, and W. T. Strunz, Phys. Rev. Lett. 86, 2913 (2001).

[14] W. T. Strunz, F. Haake, and D. Braun, Phys. Rev. A 67, 022101 (2003).

[15] W. T. Strunz and F. Haake, Phys. Rev. A 67, 022102 (2003).

[16] V. Yukalov, Ann. Phys. 327, 253 (2012).

[17] J. K. Korbicz, P. Horodecki, and R. Horodecki, Phys. Rev. Lett. 112, 120402 (2014).

[18] R. Horodecki, J. K. Korbicz, and P. Horodecki, Phys. Rev. A 91, 032122 (2015).

[19] J. Tuziemski and J. K. Korbicz, EPL 112, 40008 (2015).

[20] J. Tuziemski and J. K. Korbicz, J. Phys. A 49, 445301 (2016).

[21] P. Mironowicz, J. Korbicz, and P. Horodecki, Phys. Rev. Lett. in press; arXiv e-prints (2016), 1607.02478.

[22] S. Popescu, A. J. Short, and A. Winter, Nat. Phys. 2, 754 (2006).

[23] L. Masanes, A. J. Roncaglia, and A. Acín, Phys. Rev. E 87, 032137 (2013).

[24] F. G. S. L. Brandão, P. Ćwikliński, M. Horodecki, P. Horodecki, J. K. Korbicz, and M. Mozrzymas, Phys.
Rev. E 86, 031101 (2012).

[25] M. L. Mehta, Random matrices (Academic Press, Boston, 1991).

[26] F. Haake, Quantum Signatures of Chaos (Springer-Verlag, Berlin, Heidelberg, 2010).

[27] H. Sommers and K. Życzkowski, J. Phys. A: Math. Gen. 37, 8457 (2004).

[28] G. Sewell, Rep. Math. Phys. 56, 271 (2005),

[29] A. Auffèves and P. Grangier, Found. Phys. 46, 121 (2016).

[30] J. K. Korbicz, P. Horodecki, and R. Horodecki, Phys. Rev. A 86, 042319 (2012).

[31] H. Barnum, C. M. Caves, C. A. Fuchs, R. Jozsa, and B. Schumacher, Phys. Rev. Lett. 53, 2818 (1996).

[32] M. Piani, P. Horodecki, and R. Horodecki, Phys. Rev. Lett. 100, 090502 (2008).

[33] W. J. Hoeffding, J. Amer. Statist. Assoc. 58, 713 (1963).

[34] T. Gorin, C. Pineda, H. Kohler, and T. H. Seligman, New J. Phys. 10, 115016 (2008).

[35] M. Carrera, T. Gorin, and T. H. Seligman, Phys. Rev. A 90, 022107 (2014).

[36] R. Jozsa, J. Mod. Opt. 41, 2315 (1994).

[37] J. A. Miszczak, Z. Puchała, P. Horodecki, A. Uhlmann, and K. Życzkowski, Quantum Info. Comput. 9, 103 (2009).

[38] See Appendix.

[39] For pure $\rho_{0}$ both fidelity and decoherence factor become equal so that vanishing of the latter already implies an SBS formation.

[40] V. Giovannetti, S. Lloyd, and L. Maccone, Nat. Photonics 5, 222 (2011).

[41] The plots for a small $N_{f} / d$, Fig. 11(a), (b), show a characteristic non-monotonic behavior, which is a signature of non-Markovianity of the evolution; see e.g. H.-P. Breuer, E.-M. Laine, and J. Piilo, Phys. Rev. Lett. 103, 210401 (2009); D. Chruściński and S. Maniscalco, Phys. Rev. Lett. 112, 120404 (2014).

[42] Z. Puchała, Ł. Pawela, and K. Życzkowski, Phys. Rev. A 93, 062112 (2016).

[43] G. W. Anderson, A. Guionnet, and O. Zeitouni, An introduction to random matrices, (Cambridge University Press, Cambridge, 2010).

[44] A. Perelomov, Generalized Coherent States And Their Applications (Springer-Verlag, Berlin, 1986).

[45] A. Wünsche, Journal of Physics A: Mathematical and General 31, 8267 (1998). 


\section{Appendix A: Ensemble average over the apparatus}

\section{Average over the Haar distributed unitary transformations $U$}

In this Section we average the decoherence and the super-fidelity factors over the Haar measure. Due to the assumed independent identical distribution (i.i.d.) of the apparatus observables $B_{k}, k=1, \ldots, N$, it is enough to calculate the averages over a single observable only. This is what we shall calculate, neglecting for brevity the index $k$. We start with the decoherence factor and prove that:

Theorem 4. The decoherence factor for the single copy of the environment average over the Haar distributed unitary transformations $U$ is equal to:

$$
\left\langle\Gamma_{a a^{\prime}}(t)\right\rangle_{\mathcal{U}}=|\operatorname{Tr} D|^{2} \frac{d-\operatorname{Tr}\left[\rho_{0}^{2}\right]}{d\left(d^{2}-1\right)}+\frac{d \operatorname{Tr}\left[\rho_{0}^{2}\right]-1}{d^{2}-1}
$$

where $d$ is the local dimension of the environment.

We first write the decoherence factor as:

$$
\begin{aligned}
\Gamma_{a a^{\prime}}(t) & =\left|\operatorname{Tr}\left[\mathrm{e}^{-i\left(a-a^{\prime}\right) B t} \rho_{0}\right]\right|^{2}=\operatorname{Tr}\left[\mathrm{e}^{-i\left(a-a^{\prime}\right) B t} \rho_{0}\right] \operatorname{Tr}\left[\mathrm{e}^{-i\left(a-a^{\prime}\right) B t} \rho_{0}\right]^{\dagger}=\operatorname{Tr}\left[\mathrm{e}^{-i\left(a-a^{\prime}\right) B t} \rho_{0}\right] \operatorname{Tr}\left[\left(\mathrm{e}^{-i\left(a-a^{\prime}\right) B t}\right)^{\dagger} \rho_{0}\right] \\
& =\operatorname{Tr}\left[U D U^{\dagger} \rho_{0} \otimes U D^{\dagger} U^{\dagger} \rho_{0}\right]=\operatorname{Tr}\left[\left(U^{\dagger} \otimes U^{\dagger}\right)\left(\rho_{0} \otimes \rho_{0}\right)(U \otimes U)\left(D \otimes D^{\dagger}\right)\right]
\end{aligned}
$$

where we diagonalized the observable $B$ as $B=U \operatorname{diag}\left[\lambda_{1}, \ldots, \lambda_{d}\right] U^{+}$and defined:

$$
D \equiv \operatorname{diag}\left[\mathrm{e}^{-i \Delta_{t} \lambda_{1}}, \ldots, \mathrm{e}^{-i \Delta_{t} \lambda_{d}}\right], \quad \Delta_{t} \equiv\left(a-a^{\prime}\right) t .
$$

We also used $\operatorname{Tr} A \operatorname{Tr} B=\operatorname{Tr}(A \otimes B)$ in the second line and the following fact in the first step:

Fact 1. For any operator $X$ the following is true

$$
|\operatorname{Tr} X|^{2}=\operatorname{Tr} X \operatorname{Tr} X^{\dagger}
$$

where + stands for hermitian conjugation.

Proof.

$$
|\operatorname{Tr} X|^{2}=\operatorname{Tr} X \overline{\operatorname{Tr} X}=\operatorname{Tr} X \overline{\operatorname{Tr} X^{T}}=\operatorname{Tr} X \operatorname{Tr} X^{\dagger},
$$

where $\bar{X}$ stands for the complex conjugation of $X$ and we used $\operatorname{Tr} X=\operatorname{Tr} X^{T}$, and $\overline{X^{T}}=X^{\dagger}$.

We will also need two more well known facts:

Fact 2. For any operators $A, B$ and the SWAP operator $\mathbb{V}$, we have that:

$$
\operatorname{Tr}[\mathbb{V} A \otimes B]=\operatorname{Tr}(A B) .
$$

Proof. Let us write the SWAP operator as:

$$
\mathbb{V}=\sum_{i j}|i j\rangle\langle j i|
$$

Inserting (A7) into (A6) we have that:

$$
\begin{aligned}
\operatorname{Tr}[\mathbb{V} A \otimes B] & =\operatorname{Tr}\left(\sum_{i j}|i j\rangle\langle j i| A \otimes B\right)=\operatorname{Tr}\left(\sum_{i j}\langle j i|A \otimes B| i j\rangle\right) \\
& =\sum_{i j k l}\langle j i|A| k l\rangle\langle k l|B| i j\rangle=\operatorname{Tr}(A B) .
\end{aligned}
$$


Fact 3. For any hermitian operator $X$ from $\mathbb{C}^{d}$ to $\mathbb{C}^{d}$, it holds:

$$
\int \mathrm{d} \mathcal{U} U \otimes U X \otimes X U^{\dagger} \otimes U^{\dagger}=\frac{2}{d(d+1)} \operatorname{Tr}\left[\Pi_{s y m} X \otimes X\right] \Pi_{s y m}+\frac{2}{d(d-1)} \operatorname{Tr}\left[\Pi_{\text {asym }} X \otimes X\right] \Pi_{\text {asym }},
$$

where $\Pi_{\text {sym }}$ and $\Pi_{\text {asym }}$ are the orthogonal projectors onto the symmetric and antisymmetric subspaces, respectively, equal to

$$
\Pi_{\text {sym }} \equiv \frac{\mathbb{I}+\mathbb{V}}{2}, \quad \Pi_{\text {asym }} \equiv \frac{\mathbb{I}-\mathbb{V}}{2},
$$

where $\mathbb{V}$ is the SWAP operator.

We now integrate Eq. (A2) over $U \otimes U$. Using linearity of the trace we pull the integral inside the trace:

$$
\left\langle\Gamma_{a a^{\prime}}(t)\right\rangle_{\mathcal{U}}=\int \mathrm{d} \mathcal{U} \operatorname{Tr}\left(U^{\dagger} \otimes U^{\dagger} \rho_{0} \otimes \rho_{0} U \otimes U D \otimes D^{\dagger}\right)=\operatorname{Tr}\left[\left(\int \mathrm{d} \mathcal{U} U \otimes U \rho_{0} \otimes \rho_{0} U^{\dagger} \otimes U^{\dagger}\right) D \otimes D^{\dagger}\right]
$$

We then use Fact 3 with $X \equiv \rho_{0}$. We can easily calculate $\operatorname{Tr}\left[\Pi_{\text {sym }} \rho_{0} \otimes \rho_{0}\right]$ and $\operatorname{Tr}\left[\Pi_{\text {asym }} \rho_{0} \otimes \rho_{0}\right]$ using Facts 1 and 2 and obtain:

$$
\operatorname{Tr}\left[\Pi_{\text {sym }} \rho_{0} \otimes \rho_{0}\right] \Pi_{\text {sym }}=\frac{1}{2} \operatorname{Tr}\left[(\mathbb{I}+\mathbb{V})\left(\rho_{0} \otimes \rho_{0}\right)\right] \frac{\mathbb{I}+\mathbb{V}}{2}=\frac{1+\operatorname{Tr}\left[\rho_{0}^{2}\right]}{2} \frac{\mathbb{I}+\mathbb{V}}{2},
$$

and for the antisymmetric projector

$$
\operatorname{Tr}\left[\Pi_{\text {asym }} \rho_{0} \otimes \rho_{0}\right] \Pi_{\text {asym }}=\frac{1}{2} \operatorname{Tr}\left[(\mathbb{I}-\mathbb{V})\left(\rho_{0} \otimes \rho_{0}\right)\right] \frac{\mathbb{I}-\mathbb{V}}{2}=\frac{1-\operatorname{Tr}\left[\rho_{0}^{2}\right]}{2} \frac{\mathbb{I}-\mathbb{V}}{2} .
$$

We then again use Facts 1 and 2 to calculate the remaining traces $\operatorname{Tr}\left[(\mathbb{I} \pm \mathbb{V}) D \otimes D^{\dagger}\right]$, keeping in mind that $D$ is hermitian and that $\operatorname{Tr} D^{2}=d$. This finally gives:

$$
\left\langle\Gamma_{a a^{\prime}}(t)\right\rangle_{\mathcal{U}}=|\operatorname{Tr} \hat{D}|^{2} \frac{d-\operatorname{Tr}\left[\rho_{0}^{2}\right]}{d\left(d^{2}-1\right)}+\frac{d \operatorname{Tr}\left[\rho_{0}^{2}\right]-1}{d^{2}-1},
$$

proving our Theorem.

Using the same technique, one can also calculate the average of the super-fidelity factor. The only non-trivial part is the Hilbert-Schmidt product between the apparatus states $\rho_{a}(t)$ and $\rho_{a^{\prime}}(t)$. Using the same notation as in Eq. (A2) we obtain:

$\operatorname{Tr}\left(\rho_{a}(t) \rho_{a^{\prime}}(t)\right)=\operatorname{Tr}\left[\rho_{0} \mathrm{e}^{i\left(a-a^{\prime}\right) B t} \rho_{0} \mathrm{e}^{-i\left(a-a^{\prime}\right) B t}\right]=\operatorname{Tr}\left[U^{\dagger} \rho_{0} U D^{\dagger} U^{\dagger} \rho_{0} U D\right]=\operatorname{Tr}\left[\mathbb{V}\left(U^{\dagger} \otimes U^{\dagger}\right)\left(\rho_{0} \otimes \rho_{0}\right)(U \otimes U)\left(D^{\dagger} \otimes D\right)\right]$,

where in the last step we used Fact 2 We note that the only difference between the above Hilbert-Schmidt factor and the decoherence factor (A2) is the presence of the SWAP operator $\mathbb{V}$. Repeating the same steps as above gives:

$$
\left\langle G_{a a^{\prime}}(t)\right\rangle_{\mathcal{U}}=S_{\text {lin }}\left(\rho_{0}\right)+\frac{d-\operatorname{Tr} \rho_{0}^{2}}{d^{2}-1}+|\operatorname{Tr} D|^{2} \frac{d \operatorname{Tr} \rho_{0}^{2}-1}{d\left(d^{2}-1\right)} .
$$

Finally, we evaluate $|\operatorname{Tr} D|^{2}$ from its definition in Eq. (A3):

$$
|\operatorname{Tr} D|^{2} \equiv d+2 f_{t}(\boldsymbol{a}, \boldsymbol{\lambda}), \quad f_{t}(\boldsymbol{a}, \boldsymbol{\lambda}) \equiv \sum_{m} \sum_{n>m} \cos \left[\Delta_{t}\left(\lambda_{n}-\lambda_{m}\right)\right]=\sum_{m} \sum_{n>m} \cos \left[\left(a-a^{\prime}\right)\left(\lambda_{n}-\lambda_{m}\right) t\right],
$$

which is a function of the eigenvalues $a$ of the observable $A$ and the eigenvalues $\lambda$ of $B$. 


\section{Averaging over the eigenvalues}

After averaging over the unitary group in Sec. A1, we perform the average over the GUE eigenvalue distribution:

$$
P_{\text {gue }}(\boldsymbol{\lambda})=\frac{1}{Z} e^{-\frac{1}{2} \eta_{E} \sum_{m} \lambda_{m}^{2}} \prod_{i<j}\left|\lambda_{j}-\lambda_{i}\right|^{2}
$$

Here $\eta_{E}$ is the eigenvalue scale of the observable $B$ and $Z$ is a normalization constant (the GUE partition function). The task then is to find the following average:

$$
\left\langle f_{t}(\boldsymbol{a}, \boldsymbol{\lambda})\right\rangle=\left\langle\sum_{i} \sum_{j>i} \cos \left[\Delta_{t}\left(\lambda_{i}-\lambda_{j}\right)\right]\right\rangle=\sum_{i} \sum_{j>i} \int d \boldsymbol{\lambda} P_{\text {gue }}(\boldsymbol{\lambda}) \cos \left[\Delta_{t}\left(\lambda_{i}-\lambda_{j}\right)\right]
$$

Quite surprisingly, this average can be performed explicitly using the standard methods of dealing with GUE [25]. We first introduce the harmonic oscillator wave functions:

$$
\phi_{n}(x) \equiv \frac{1}{\sqrt{\sqrt{2 \pi} n !}} e^{-\frac{x^{2}}{4}} H e_{n}(x) .
$$

Notice that we define the wave functions using the so-called "probabilist" Hermite polynomials:

$$
H e_{n}(x) \equiv(-1)^{n} e^{\frac{1}{2} x^{2}} \frac{d^{n}}{d x^{n}} e^{-\frac{1}{2} x^{2}}
$$

That is, they are orthogonal with respect to the weight function $\exp \left[-x^{2} / 2\right]$, and are related to the physicist's polynomials $H_{n}(x)$ via $H e_{n}(x)=2^{-n / 2} H_{n}(x / \sqrt{2})$. Of course we still have $\int d x \phi_{n}(x) \phi_{m}(x)=\delta_{n m}$. Then the GUE eigenvalue distribution takes on a very compact and elegant form, after rescaling $\lambda_{k} \equiv \frac{\zeta_{k}}{\sqrt{\eta_{E}}}$ [25] (6.2.4):

$$
P_{g u e}(\lambda) d \boldsymbol{\lambda}=\frac{1}{d !} \operatorname{det}\left[\phi_{j-1}\left(\zeta_{i}\right)\right]^{2} d \zeta
$$

where $i, j=1, \ldots, d$.

\section{a. Exploiting the symmetry}

A crucial step is the realization that this average has an index permutation symmetry. Let $\sigma \in S_{d}$, be a permutation, then:

$$
P_{\text {gue }}\left(\lambda_{\sigma(1)}, \ldots, \lambda_{\sigma(d)}\right)=P_{\text {gue }}\left(\lambda_{1}, \ldots, \lambda_{d}\right) .
$$

Analogously we have (keeping the eigenvalues $\boldsymbol{a}$ fixed):

$$
f_{t}\left(\boldsymbol{a}, \lambda_{\sigma(1)}, \ldots, \lambda_{\sigma(d)}\right)=\sum_{i<j} \cos \left[\Delta_{t}\left(\lambda_{\sigma(i)}-\lambda_{\sigma(j)}\right)\right]=\sum_{i<j} \cos \left[\Delta_{t}\left(\lambda_{i}-\lambda_{j}\right)\right]=f_{t}(\boldsymbol{a}, \boldsymbol{\lambda}) .
$$

This is because in both expressions the eigenvalue functions are symmetric and all pairs of indices are taken (i.e. the product or sum is over all $i<j$ ). Equivalently, we can recall that $\operatorname{Tr} D=\sum_{i} \mathrm{e}^{-\mathrm{i} \Delta_{t} \lambda_{i}}$ and from Eq. (A17) $f_{t}(\boldsymbol{a}, \boldsymbol{\lambda})=1 / 2\left(|\operatorname{Tr} D|^{2}-d\right)$, which is clearly symmetric under the permutations. Hence, the calculation of the average $f_{t}(\boldsymbol{a}, \boldsymbol{\lambda})$ reduces to a single term:

$$
\left\langle f_{t}(\boldsymbol{a}, \boldsymbol{\lambda})\right\rangle=\frac{d(d-1)}{2} \int d \boldsymbol{\lambda} P_{g u e}(\boldsymbol{\lambda}) \cos \left[\Delta_{t}\left(\lambda_{1}-\lambda_{2}\right)\right]
$$


As the integrand only depends on two variables, we can take the marginal distribution, which is essentially the 2-point correlation function, defined as [25] (6.1.2):

$$
R_{2}\left(\lambda_{1}, \lambda_{2}\right) \equiv \frac{d !}{(d-2) !} \int d \lambda_{3} \cdots d \lambda_{d} P_{g u e}\left(\lambda_{1}, \ldots, \lambda_{d}\right)
$$

Hence, we have reduced the problem to the integral:

$$
\left\langle f_{t}(\boldsymbol{a}, \boldsymbol{\lambda})\right\rangle=\frac{1}{2} \int d \lambda_{1} d \lambda_{2} R_{2}\left(\lambda_{1}, \lambda_{2}\right) \cos \left[\Delta_{t}\left(\lambda_{1}-\lambda_{2}\right)\right]
$$

\section{b. The crucial integral}

To calculate A26 we will need the following integral:

$$
J_{n, m}(\alpha) \equiv \int d x \phi_{n}(x) \phi_{m}(x) e^{\mathrm{i} \alpha x} .
$$

In fact A27) may be interpreted as a special case of a matrix element of the displacement operator $D(\beta)=$ $\exp \left[\beta \hat{a}^{\dagger}-\beta^{*} \hat{a}\right]$ in the Fock basis $\{|n\rangle\}_{n \in \mathbb{N}}$. We recover our integral setting $\beta \equiv \mathbf{i} \alpha, \alpha \in \mathbb{R}$. This turns out to be a well known quantity in quantum optics (see e.g. [44]), but for completeness we present its calculation below. Without a loss of generality, we will assume $m \geq n$. First, we express the wavefunctions through the (probabilist) Hermite polynomials as in Eq. (A20) and use the generating function for $\mathrm{He}_{n}(x)$ with parameters $r, s$ to perform the integral:

$$
\begin{aligned}
J_{n, m}(\alpha) & =\frac{1}{\sqrt{2 \pi n ! m !}} \int d x H e_{n}(x) H e_{m}(x) e^{-\frac{1}{2} x^{2}+\mathbf{i} \alpha x} \\
& =\left.\left.\frac{1}{\sqrt{2 \pi n ! m !}} \frac{\partial^{n}}{\partial r^{n}}\right|_{r=0} \frac{\partial^{m}}{\partial s^{m}}\right|_{s=0} \int d x e^{(\mathbf{i} \alpha+r+s) x-\frac{1}{2}\left(r^{2}+s^{2}+x^{2}\right)} \\
& =\left.\left.\frac{e^{-\frac{1}{2} \alpha^{2}}}{\sqrt{n ! m !}} \frac{\partial^{n}}{\partial r^{n}}\right|_{r=0} \frac{\partial^{m}}{\partial s^{m}}\right|_{s=0} e^{\mathbf{i} \alpha r+\mathbf{i} \alpha s+r s} \\
& =\left.\frac{e^{-\frac{1}{2} \alpha^{2}}}{\sqrt{n ! m !}} \frac{\partial^{n}}{\partial r^{n}}\right|_{r=0}(\mathbf{i} \alpha+r)^{m} e^{\mathbf{i} \alpha r}
\end{aligned}
$$

Then we use the binomial formula for the derivatives $\frac{d^{n}}{d x^{n}} f(x) g(x)=\sum_{k=0}^{n}\left(\begin{array}{l}n \\ k\end{array}\right) \frac{d^{n-k} f(x)}{d x^{n-k}} \frac{d^{k} g(x)}{d x^{k}}$. Since $m \geq n$ we don't run into any unexpected problems and obtain:

$$
J_{n, m}(\alpha)=\frac{e^{-\frac{1}{2} \alpha^{2}}}{\sqrt{n ! m !}} \sum_{k=0}^{n}\left(\begin{array}{l}
n \\
k
\end{array}\right)\left(\begin{array}{l}
m \\
k
\end{array}\right) k !(\mathbf{i} \alpha)^{n+m-2 k}
$$

This may be nicely expressed in terms of the associated Laguerre polynomials as (taking $\alpha \in \mathbb{R}$ ):

$$
J_{n, m}(\alpha)=e^{-\frac{1}{2} \alpha^{2}} \sqrt{\frac{n !}{m !}}(\mathbf{i} \alpha)^{m-n} L_{n}^{(m-n)}\left(\alpha^{2}\right),
$$

where:

$$
L_{n}^{(m)}(x) \equiv \sum_{k=0}^{n}\left(\begin{array}{c}
n+m \\
n-k
\end{array}\right) \frac{(-x)^{k}}{k !}
$$

(we adopt the common standardization for the Laguerre polynomials that the leading coefficient is equal to $\left.(-1)^{n} / n !\right)$. Eq. A27) can also be expressed more compactly in terms of the, so-called, 2D Laguerre functions introduced in [45]:

$$
\langle m|D(\alpha)| n\rangle=(-1)^{n} \sqrt{\pi} l_{m, n}\left(\alpha, \alpha^{*}\right)
$$


for a general complex displacement $\alpha$. The 2D Laguerre functions are defined as [45]:

$$
l_{m, n}\left(z, z^{*}\right) \equiv \frac{1}{\sqrt{\pi}} e^{-\frac{z z^{*}}{2}} \frac{1}{\sqrt{m ! n !}} \sum_{j=0}^{m}\left(\begin{array}{c}
m \\
j
\end{array}\right)\left(\begin{array}{c}
n \\
j
\end{array}\right) j !(-1)^{j} z^{m-j} z^{* n-j} .
$$

\section{c. Putting the results together}

We return to calculating the integral (A26). We use Eq. A21], rescale the variables, and introduce a more friendly notation $(x, y) \equiv\left(\zeta_{1}, \zeta_{2}\right)$ :

$$
\left\langle f_{t}(\boldsymbol{a}, \boldsymbol{\lambda})\right\rangle=\frac{1}{2} \int d x d y R_{2}(x, y) \cos \left[\tilde{\Delta}_{t}(x-y)\right],
$$

where:

$$
\tilde{\Delta}_{t} \equiv \frac{\left(a-a^{\prime}\right) t}{\sqrt{\eta_{E}}} .
$$

Now, Dyson's Theorem will let us calculate the 2-point correlation function [25] Thm 5.14, (6.2.6-7):

$$
R_{2}(x, y)=K(x, x) K(y, y)-K(x, y)^{2},
$$

where the kernel is defined through the oscillator wave-functions (A20) as:

$$
K(x, y) \equiv \sum_{j=0}^{d-1} \phi_{j}(x) \phi_{j}(y)
$$

Hence, we can express our integral as the following sum:

$$
\left\langle f_{t}(\boldsymbol{a}, \boldsymbol{\lambda})\right\rangle=\sum_{n=0}^{d-1} \sum_{m=0}^{d-1} \int d x d y\left(\phi_{n}(x)^{2} \phi_{m}(y)^{2}-\phi_{n}(x) \phi_{m}(x) \phi_{n}(y) \phi_{m}(y)\right) \cos \left[\tilde{\Delta}_{t}(x-y)\right] .
$$

By expressing the cosine function in exponential form, the integrals become separable and we obtain:

$$
\left\langle f_{t}(\boldsymbol{a}, \boldsymbol{\lambda})\right\rangle=\frac{1}{4} \sum_{n=0}^{d-1} \sum_{m=0}^{d-1}\left(A_{n, m}+\tilde{A}_{n, m}-2 B_{n, m}\right),
$$

where we introduced auxiliary functions:

$$
\begin{aligned}
A_{n, m} & \equiv J_{n, n}\left(\tilde{\Delta}_{t}\right) J_{m, m}\left(-\tilde{\Delta}_{t}\right), \\
\tilde{A}_{n, m} & \equiv J_{n, n}\left(-\tilde{\Delta}_{t}\right) J_{m, m}\left(\tilde{\Delta}_{t}\right), \\
B_{n, m} & \equiv J_{n, m}\left(\tilde{\Delta}_{t}\right) J_{n, m}\left(-\tilde{\Delta}_{t}\right) .
\end{aligned}
$$

Now, we are able to separate the $n, m$ summation into three parts $n<m, n=m$, and $n>m$. From the definition of the auxiliary functions, we easily see that the diagonal summation $n=m$ vanishes. The remaining sums $n<m$ and $n>m$ become the same, since $A_{m, n}=\tilde{A}_{n, m}$ and $B_{m, n}=B_{n, m}$. Hence, for convenience's sake we will calculate the sum $m>n$ only. We use the explicit result (A32) for the $J_{n, m}$ and obtain:

$$
A_{n, m}=e^{-\tilde{\Delta}_{t}^{2}} \sum_{k=0}^{n} \sum_{l=0}^{m}\left(\begin{array}{l}
n \\
k
\end{array}\right)\left(\begin{array}{c}
m \\
l
\end{array}\right) \frac{(-1)^{n+m-k-l} \tilde{\Delta}_{t}^{2(n+m-k-l)}}{(n-k) !(m-l) !} .
$$


And by doing the same for $\tilde{A}$, we indeed realize that $A_{n, m}=\tilde{A}_{n, m}$. For $B_{n, m}$, in turn, we obtain:

$$
B_{n, m}=e^{-\tilde{\Delta}_{t}^{2}} \sum_{k=0}^{n} \sum_{l=0}^{n}\left(\begin{array}{l}
n \\
k
\end{array}\right)\left(\begin{array}{c}
m \\
l
\end{array}\right) \frac{(-1)^{k+l} \tilde{\Delta}_{t}^{2(n+m-k-l)}}{(m-k) !(n-l) !} .
$$

Hence, putting it all together (with a factor of 2, since now we only sum $m>n$ ), we arrive at:

$$
\left\langle f_{t}(\boldsymbol{a}, \boldsymbol{\lambda})\right\rangle=e^{-\tilde{\Delta}_{t}^{2}} \sum_{n=0}^{d-2} \sum_{m=n+1}^{d-1}\left[\sum_{k=0}^{n} \sum_{l=0}^{m}\left(\begin{array}{l}
n \\
k
\end{array}\right)\left(\begin{array}{c}
m \\
l
\end{array}\right) \frac{(-1)^{n+m-k-l} \tilde{\Delta}_{t}^{2(n+m-k-l)}}{(n-k) !(m-l) !}-\sum_{k=0}^{n} \sum_{l=0}^{n}\left(\begin{array}{l}
n \\
k
\end{array}\right)\left(\begin{array}{c}
m \\
l
\end{array}\right) \frac{(-1)^{k+l} \tilde{\Delta}_{t}^{2(n+m-k-l)}}{(m-k) !(n-l) !}\right] .
$$

This result may also be rewritten using the associated Laguerre polynomials and Eq. (A33):

$$
\left\langle f_{t}(\boldsymbol{a}, \boldsymbol{\lambda})\right\rangle=e^{-\tilde{\Delta}_{t}^{2}} \sum_{n=0}^{d-2} \sum_{m=n+1}^{d-1}\left[L_{n}^{(0)}\left(\tilde{\Delta}_{t}^{2}\right) L_{m}^{(0)}\left(\tilde{\Delta}_{t}^{2}\right)-\frac{n !}{m !} \tilde{\Delta}_{t}^{2(m-n)}\left[L_{n}^{(m-n)}\left(\tilde{\Delta}_{t}^{2}\right)\right]^{2}\right]
$$

One of the sums can be performed using the following identity for the associated Laguerre polynomials:

$$
\sum_{m=0}^{M} L_{m}^{(\alpha)}(x)=L_{M}^{(\alpha+1)}(x)
$$

giving:

$$
\left\langle f_{t}(\boldsymbol{a}, \boldsymbol{\lambda})\right\rangle=e^{-\tilde{\Delta}_{t}^{2}}\left[L_{d-1}^{(1)}\left(\tilde{\Delta}_{t}^{2}\right) L_{d-2}^{(1)}\left(\tilde{\Delta}_{t}^{2}\right)-\sum_{n=0}^{d-2} L_{n}^{(0)}\left(\tilde{\Delta}_{t}^{2}\right) L_{n}^{(1)}\left(\tilde{\Delta}_{t}^{2}\right)-\sum_{n=0}^{d-2} \sum_{m=n+1}^{d-1} \frac{n !}{m !} \tilde{\Delta}_{t}^{2(m-n)}\left[L_{n}^{(m-n)}\left(\tilde{\Delta}_{t}^{2}\right)\right]^{2}\right] .
$$

However, we keep (A49) in the main text since it is more compact.

Please note that in the main text we do not directly use $\left\langle f_{t}(\boldsymbol{a}, \boldsymbol{\lambda})\right\rangle$, but rather separate its Gaussian and polynomial parts, i.e.

$$
\left\langle f_{t}(\boldsymbol{a}, \boldsymbol{\lambda})\right\rangle \equiv \mathrm{e}^{-\tilde{\Delta}_{t}^{2}} p\left(d, \tilde{\Delta}_{t}\right)
$$

\section{Appendix B: Short time analysis}

In this Section we perform the final averaging over the system observable $A$. In the previous Section, we have used the i.i.d. property of the apparatus ensemble to reduce the big, compound averages to single copy ones. Here, however, we cannot do so, as ultimately we are interested in the macroscopic quantities $\Gamma_{a a^{\prime}}^{N_{u n o}}, G_{a a^{\prime}}^{m a c}$. Thus we need:

$$
\left\langle\left\langle X^{f}(t)\right\rangle\right\rangle_{a a^{\prime}} \equiv \int d \boldsymbol{a} P_{g u e}(\boldsymbol{a})\left\langle X_{a a^{\prime}}(t)\right\rangle^{N_{f}},
$$

where $X_{a a^{\prime}}=\Gamma_{a a^{\prime}}$ or $G_{a a^{\prime}}$ and $f=u n o$ or mac respectively. We note that both $\left\langle\Gamma_{a a^{\prime}}(t)\right\rangle,\left\langle G_{a a^{\prime}}(t)\right\rangle$ depend on $A$ only through the eigenvalue differences $\left|a-a^{\prime}\right|$. Thus, the $A$-averaging reduces to averaging over the eigenvalues only with its own GUE eigenvalue distribution:

$$
P_{\text {gue }}(\boldsymbol{a}) \equiv P_{\text {gue }}\left(a_{1}, \ldots, a_{d_{S}}\right) \equiv \frac{1}{Z_{S}} e^{-\frac{1}{2} \eta_{S} \sum_{l} a_{l}^{2}} \prod_{i<j}\left|a_{j}-a_{i}\right|^{2}
$$

where $d_{S}$ is the system dimension and $\eta_{S}$ is the eigenvalue scale of the system observable $A$. From the permutational symmetry of the GUE distribution (best seen through the Vandermonde determinant), the integral in Eq. (B1) reduces to the integration with the same 2-point correlation function (A25), but now defined for the distribution (B2) and thus all the averages for different pairs $a a^{\prime}$ are the same and equal to:

$$
\left\langle\left\langle X^{f}(t)\right\rangle\right\rangle=\left[\frac{d_{S} !}{\left(d_{S}-2\right) !}\right]^{-1} \int d a d a^{\prime} R_{2}\left(a, a^{\prime}\right)\left\langle X_{a a^{\prime}}(t)\right\rangle^{N_{f}} .
$$


The resulting integral is too complicated to be performed analytically and actually this is in fact not needed as we see from Eq. A52 that $\left\langle f_{t}(\boldsymbol{a}, \boldsymbol{\lambda})\right\rangle$ will eventually decay so that both factors will approach their noise-floor values (cf. Result 1 from the main text). What we are interested in are the relevant timescales. We can estimate lower bounds on those timescales from the decay times of (A52). We will perform this analysis in the following steps: o) assume a short-time limit; i) approximate the polynomial $p(d, \tilde{\Delta})$ of Eq. $\left(\mathrm{A} 52\right.$; ii) approximate the $N_{f}$ power; iii) using Eqs. (A39), A40), and (A20) estimate the fastest decaying term in (B3). This will then give lower bounds on the desired times of the asymptotic approach: The latter are for sure greater than the initial decay times.

First, we assume $\tilde{\Delta}_{t} \ll 1$, or $t \ll \sqrt{\eta_{E}} /\left|a-a^{\prime}\right|$. The maximum of $\left|a-a^{\prime}\right|$ is of the order of $\sqrt{d_{S} / \eta_{S}}$ from the Wigner semi-circle law, defining the short-time limit:

$$
t \ll \sqrt{\frac{\eta_{E} \eta_{S}}{d_{S}}} \equiv \frac{1}{g \sqrt{d_{S}}}
$$

where $g \equiv 1 / \sqrt{\eta_{E} \eta_{S}}$ is the effective interaction strength.

We now explicitly calculate the coefficients of the lowest order terms in $p(d, \tilde{\Delta})$ directly from Eq. A48). One immediately sees that the polynomial is even so the lowest terms are the constant and the quadratic ones. The constant term occurs when $k+l=m+n$, but this can only occur in the first summand of the polynomial when $k=n$ and $l=m$, so that we have:

$$
\sum_{n<m}[1]=\frac{d(d-1)}{2}
$$

The quadratic term occurs when the indices fulfill the condition $k+l+1=m+n$. On the first term, this can occur if $(k=n, l=m-1)$ or if $(k=n-1, l=m)$. On the second term this can occur only when the $m$ index is $m+1$ and the inner indices are $k=l=n$. Thus we obtain:

$$
\sum_{n<m}\left[m(-1) \tilde{\Delta}_{t}^{2}\right]+\sum_{n<m}\left[n(-1) \tilde{\Delta}_{t}^{2}\right]-\sum_{n}\left[(n+1) \tilde{\Delta}_{t}^{2}\right]=-\frac{d^{2}(d-1)}{2} \tilde{\Delta}_{t}^{2}
$$

Thus, for short times we have:

$$
p\left(d, \tilde{\Delta}_{t}\right)=\frac{d(d-1)}{2}\left(1-d \tilde{\Delta}_{t}^{2}\right)+O\left(\tilde{\Delta}_{t}^{4}\right) .
$$

To proceed further, we upper bound the above expression by the Gaussian function: $1-d \tilde{\Delta}_{t}^{2} \leq \mathrm{e}^{-d \tilde{\Delta}_{t}^{2}}$ resulting from Eq. A52) in the short-time bound:

$$
\left\langle f_{t}(\boldsymbol{a}, \boldsymbol{\lambda})\right\rangle \lesssim \mathrm{e}^{-(d+1) \tilde{\Delta}_{t}^{2}} \equiv \mathrm{e}^{-\left(t / \tau_{a a^{\prime}}\right)^{2}}, \quad \tau_{a a^{\prime}} \equiv \frac{\sqrt{\eta_{E}}}{\sqrt{d+1}\left|a-a^{\prime}\right|} .
$$

We then use the following approximation of the power:

$$
\left(\alpha+\beta \mathrm{e}^{-x}\right)^{N_{f}} \approx(\alpha+\beta)^{N_{f}} \mathrm{e}^{-N_{f} \frac{\beta}{\alpha+\beta} x}
$$

for $x \ll 1$. We apply it to the single-copy averaged factors:

$$
\begin{aligned}
& \left\langle\Gamma_{a a^{\prime}}(t)\right\rangle=\frac{1+\operatorname{Tr} \rho_{0}^{2}}{d+1}+\left\langle f_{t}(\boldsymbol{a}, \boldsymbol{\lambda})\right\rangle \frac{2\left(d-\operatorname{Tr} \rho_{0}^{2}\right)}{d\left(d^{2}-1\right)}, \\
& \left\langle G_{a a^{\prime}}(t)\right\rangle=S_{l i n}\left(\rho_{0}\right)+\frac{1+\operatorname{Tr} \rho_{0}^{2}}{d+1}+\left\langle f_{t}(\boldsymbol{a}, \boldsymbol{\lambda})\right\rangle \frac{2\left(d \operatorname{Tr} \rho_{0}^{2}-1\right)}{d\left(d^{2}-1\right)},
\end{aligned}
$$

identifying $\beta$ with the constant terms and $\alpha$ with the multiplicative one. After a simple algebra, this leads to the following short time approximations of the macroscopic factors:

$$
\begin{aligned}
& \left\langle\Gamma_{a a^{\prime}}^{u n o}(t)\right\rangle=\left\langle\Gamma_{a a^{\prime}}(t)\right\rangle^{N_{\text {uno }}} \approx \exp \left[-N_{\text {uno }} \tilde{\Delta}_{t}^{2}\left(d-\left\langle\operatorname{Tr} \rho_{0}^{2}\right\rangle\right)\right], \\
& \left\langle G_{a a^{\prime}}^{m a c}(t)\right\rangle=\left\langle G_{a a^{\prime}}(t)\right\rangle^{N_{m a c}} \approx \exp \left[-N_{m a c} \tilde{\Delta}_{t}^{2}\left(d\left\langle\operatorname{Tr} \rho_{0}^{2}\right\rangle-1\right)\right] .
\end{aligned}
$$


For $\left\langle\operatorname{Tr} \rho_{0}^{2}\right\rangle$ we can use either the Hilbert-Schmidt or Bures measure from the main text. It is interesting to note that in any case $\left\langle\operatorname{Tr} \rho_{0}^{2}\right\rangle \propto 1 / d$, so that (B12) shows a dependence on both $N_{\text {uno }}$ and $d$ while (B13) shows a dependence mainly on $N_{\text {mac }}$.

We are now ready to estimate the lower bound on the decay time of the integral (B3). Substituting Eq. (B12) or (B13) and using Eqs. (A39), (A40), and (A20), we are left with a sum of integrals of the following structure:

$$
\int d a d a^{\prime} H e_{m}(a) H e_{n}(a) H e_{j}\left(a^{\prime}\right) H e_{l}\left(a^{\prime}\right) \mathrm{e}^{-\mu_{t}\left(a-a^{\prime}\right)^{2}-\frac{1}{2}\left(a^{2}+a^{\prime 2}\right)} \sim \int d a d a^{\prime} a^{r} a^{\prime s} \mathrm{e}^{-\mu_{t}\left(a-a^{\prime}\right)^{2}-\frac{1}{2}\left(a^{2}+a^{\prime 2}\right),}
$$

where the powers satisfy $0 \leq r, s \leq 2\left(d_{S}-1\right)$ (cf. Eq. (A40) ) and $\mu_{t} \equiv N_{u n o}(g t)^{2}\left(d-\left\langle\operatorname{Tr} \rho_{0}^{2}\right\rangle\right)$ for the decoherence factor and $\mu_{t} \equiv N_{\operatorname{mac}}(g t)^{2}\left(d\left\langle\operatorname{Tr} \rho_{0}^{2}\right\rangle-1\right)$ for the super-fidelity (cf. Eqs. B12 B13). To separate the integrals, we diagonalize the quadratic form in the exponent. Its eigenvalues read $\left(1+4 \mu_{t}\right) / 2$ and $1 / 2$. Denoting by $O$ the diagonalizing $S O(2)$ transformation, we pass to the new variables $(x, y)^{T} \equiv O\left(a, a^{\prime}\right)^{T}$ in which the integrals (B14) separate:

$$
\int d a d a^{\prime} a^{r} a^{\prime s} \mathrm{e}^{-\mu_{t}\left(a-a^{\prime}\right)^{2}-\frac{1}{2}\left(a^{2}+a^{\prime 2}\right)} \sim\left(\int d x x^{\gamma} \mathrm{e}^{-\frac{1+4 \mu_{t}}{2} x^{2}}\right)\left(\int d y y^{\delta} \mathrm{e}^{-\frac{1}{2} y^{2}}\right),
$$

where $0 \leq \gamma, \delta \leq 2\left(d_{S}-1\right), \gamma+\delta=r+s$. The integrals on the right hand side are now elementary and depend on time as $1 /\left(1+4 \mu_{t}\right)^{\gamma+\epsilon}$, where $\epsilon=1$ or $1 / 2$ depending on the parity of the power $\gamma$. Putting all together, the integral (B3) has the following time dependence (we neglect possible time dependent coefficients, originating from the matrix $O$, as they are given by the sine and cosine functions and hence $\sim O(1)$ ):

$$
\left\langle\left\langle X^{f}(t)\right\rangle\right\rangle \sim \frac{1}{\sqrt{1+4 \mu_{t}}}+\frac{1}{1+4 \mu_{t}}+\frac{1}{\left(1+4 \mu_{t}\right)^{3 / 2}}+\cdots+\frac{1}{\left(1+4 \mu_{t}\right)^{2\left(d_{s}-1\right)+\epsilon}} .
$$

The fastest decaying is the last term, which for $\mu_{t} \ll 1$ or $t \ll O\left(\frac{1}{\sqrt{g^{2} N_{f}}}\right)$ can be approximated by an exponential:

$$
\frac{1}{\left(1+4 \mu_{t}\right)^{2\left(d_{S}-1\right)+\epsilon}} \approx \exp \left[-8 d_{S} c(d) N_{f}(g t)^{2}\right] \text {, }
$$

where we neglected the factor $-2+\epsilon=-1$ or $-3 / 2$ in the power as compared to $2 d_{S}$ and $c(d) \equiv d-\left\langle\operatorname{Tr} \rho_{0}^{2}\right\rangle$ for the decoherence factor and $c(d) \equiv d\left\langle\operatorname{Tr} \rho_{0}^{2}\right\rangle-1$ for the super-fidelity. This finally gives the following lower bounds on the decay times:

$$
\begin{gathered}
\tau_{\text {dec }} \equiv\left[8 g^{2} N_{\text {uno }} d_{S}\left(d-\left\langle\operatorname{Tr} \rho_{0}^{2}\right\rangle\right)\right]^{-1 / 2} \sim\left[8 g^{2} N_{\text {uno }} d_{S} d\right]^{-1 / 2}, \\
\tau_{f i d} \equiv\left[8 g^{2} N_{\text {mac }} d_{S}\left(d\left\langle\operatorname{Tr} \rho_{0}^{2}\right\rangle-1\right)\right]^{-1 / 2} \sim\left[8 g^{2} N_{\text {mac }} d_{S}\right]^{-1 / 2},
\end{gathered}
$$

where the simplified estimates are in the limit of a large local dimension of the apparatus $d$. The above times are clearly within the short-time approximation range (B4). It is interesting to note that the above separation of physical time-scales of decoherence and information accumulation is, on the mathematical level, a consequence of a simple symmetry difference in the initial formulas A2 and A15. The latter has an additional SWAP operator $\mathbb{V}$ under the trace.

As mentioned, the above timescales are only lower bounds for the actual times of the noise floor approach. Perhaps they can be tightened using different analytical tools, but for the purpose of this work we will use the above $\tau_{\text {dec }}, \tau_{\text {fid }}$.

\section{Appendix C: Upperbounding the exact expression for $\left\langle f_{t}(\boldsymbol{a}, \lambda)\right\rangle$}

This Section is complimentary to the main line of reasoning and is not necessary for understanding it. The exact expression (A48) is algebraically complicated and a simplified expression, reproducing short- and long-time behavior and preferably upper bounding it would be desirable. Here we attempt a construction of such an upper bound. For short times, we may neglect in the average $\left\langle f_{t}(\boldsymbol{a}, \boldsymbol{\lambda})\right\rangle=p\left(d, \tilde{\Delta}_{t}\right) \mathrm{e}^{-\tilde{\Delta}_{t}^{2}}$ all terms except the first two giving 


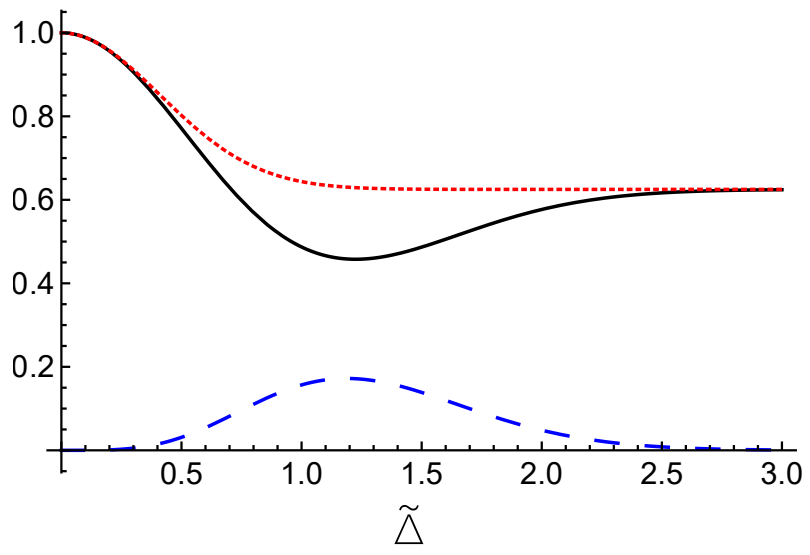

(a) $d=2$

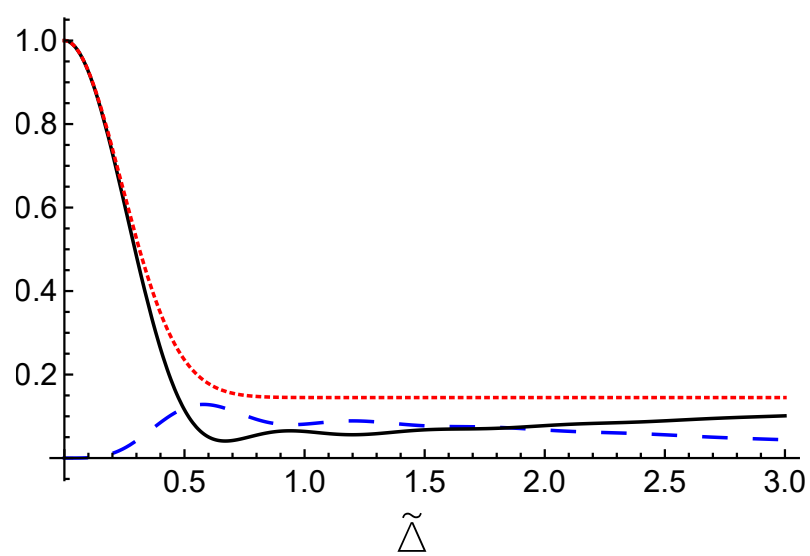

(c) $d=8$

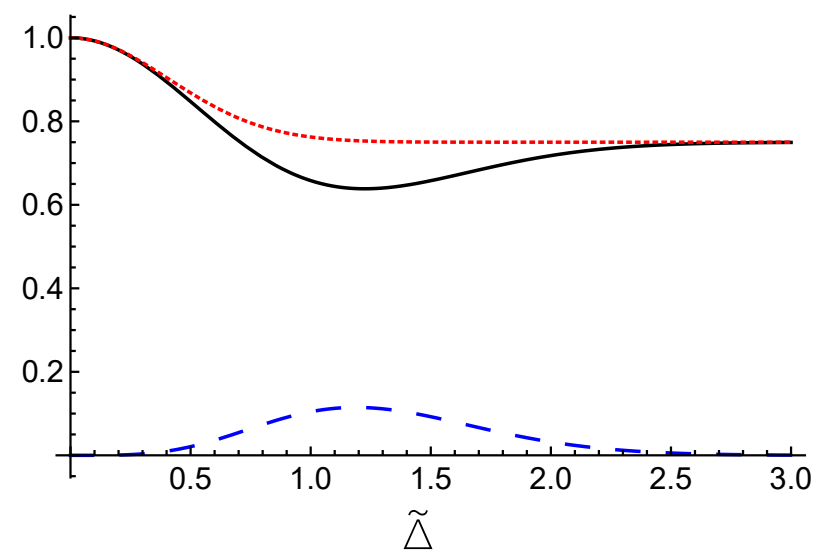

(b) $d=2$

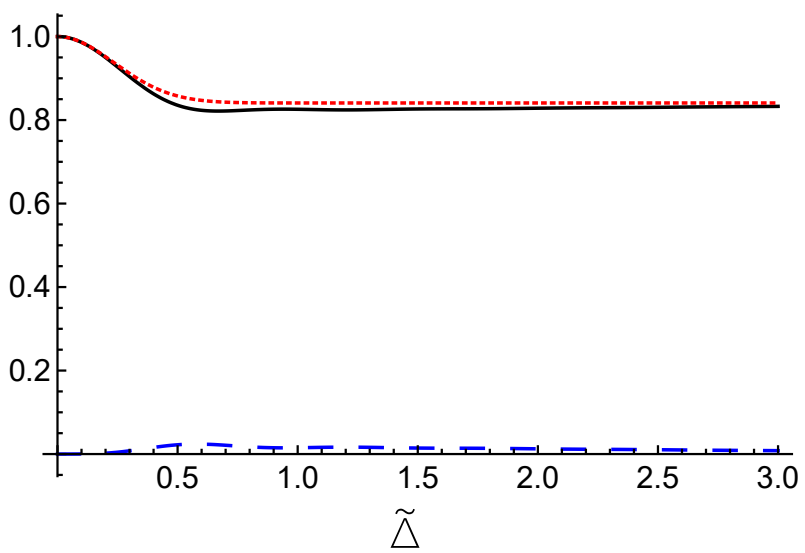

(d) $d=8$

FIG. B1: (Color online). In figures (a) and (c) the dotted (red) curve represents the Ansatz $\tilde{\Gamma}(t)$, the solid (black) line represents the exact decoherence factor $\langle\Gamma(t)\rangle$, and the dashed (blue) curve the difference $\tilde{\Gamma}(t)-\langle\Gamma(t)\rangle$. Similarly, in (b) and (d) the dotted (red) curve represents the Ansatz $\tilde{G}(t)$, the solid (black) line represents the exact averaged super-fidelity $\langle G(t)\rangle$, and the dashed (blue) curve the difference $\tilde{G}(t)-\langle G(t)\rangle$. The timescale is given in units of $\tilde{\Delta}=\left(a-a^{\prime}\right) / \sqrt{\eta_{E}}$, the Bures average purity is used throughout, and the exact integration is done assuming $d_{S}=2$.

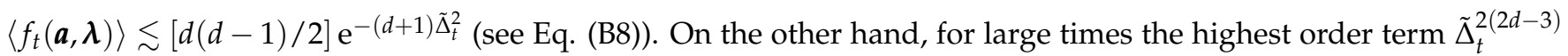
dominates and it always comes with a negative coefficient as can be seen from Eq. (A48). These observations suggest the following Ansatz:

$$
\begin{aligned}
& \tilde{\Gamma}_{a a^{\prime}}(t) \equiv \frac{1+\left\langle\operatorname{Tr} \rho_{0}^{2}\right\rangle}{d+1}+\frac{d-\left\langle\operatorname{Tr} \rho_{0}^{2}\right\rangle}{d+1} \mathrm{e}^{-(d+1) \tilde{\Delta}_{t}^{2},} \\
& \tilde{G}_{a a^{\prime}}(t) \equiv\left\langle S_{\operatorname{lin}}\left(\rho_{0}\right)\right\rangle+\frac{1+\left\langle\operatorname{Tr} \rho_{0}^{2}\right\rangle}{d+1}+\frac{d\left\langle\operatorname{Tr} \rho_{0}^{2}\right\rangle-1}{d+1} \mathrm{e}^{-(d+1) \tilde{\Delta}_{t}^{2},}
\end{aligned}
$$

for the decoherence and the superfidelity factors respectively. By construction, the expressions (C1|C2) reproduce the correct short time behavior as $\left\langle\Gamma_{a a^{\prime}}(t)\right\rangle=\tilde{\Gamma}_{a a^{\prime}}(t)+O\left(\tilde{\Delta}_{t}^{4}\right)$, and similarly $\left\langle G_{a a^{\prime}}(t)\right\rangle$, for $t \ll \tau_{a a^{\prime}}$, where $\tau_{a a^{\prime}}$ is the characteristic time of the Gaussian decay in $(\mathrm{C} 1 \mid \mathrm{C} 2)$ :

$$
\tau_{a a^{\prime}} \equiv \frac{\sqrt{\eta_{E}}}{\sqrt{d+1}\left|a-a^{\prime}\right|} .
$$

They are also upper bounds since $1-x \leq \mathrm{e}^{-x}$ (cf. (B7)). Similarly, for long times $t \gg \tau_{a a^{\prime}}$, C1 C2 reproduce the correct white noise factors and also upper bound the exact averages, since $p\left(d, \tilde{\Delta}_{t}\right)$ is then negative. These two facts together suggest that $[\mathrm{C} 1 \mid \mathrm{C} 2)$ may be upper bounds for all times $t$ and dimensions $d$. Unfortunately we 
were unable to prove it analytically, but numerical evidence for $d \leq 20$ suggests that it is indeed so. In Fig. B1.we present some sample plots of both the exact expressions and $[\mathrm{C} 1 \mid \mathrm{C} 2$ together with the errors. The price to pay for working with the simplified expressions (C1]C2) is that one loses the important physical information on nonMarkovianity, reflected by the non-monotonic behaviour of the exact averages for low rations $\mathrm{N} / d$. Also the exact functions approach their asymptotic limits from below, signalizing recovery of coherences/loss of information in the environment, while (C1) C2 approach them from above. However, if one is solely interested in an SBS formation, an upper bound decaying to the correct noise level is just enough. 